\title{
Experimental Study on Neural Feedback in Embedded System Teaching Processing Based on ERP Signal Analysis
}

\author{
https://doi.org/10.3991/ijet.v14i12.10715 \\ Danbing Du $(\bowtie)$ \\ Changchun Normal University education department, Changchun, China \\ 44093945 dqq. com
}

\begin{abstract}
Embedded system teaching is an indispensable part in the process of computer language learning. This paper analyzes the neural feedback of embedded system learners in learning embedded systems by observing the generation and fluctuation of EEG signals. The ERP signals of computer learners in learning embedded system are monitored by ERP technology. The results show that the embedded system leads to the changes in EEG of computer linguists, and the changes of EEG amplitudes caused by different embedded systems. Combined with previous studies, it is shown that the embedded system processing has different effects on neural feedback. The analysis of EEG signals provides some theoretical basis and new ideas for embedded system teaching.
\end{abstract}

Keywords-EEG, embedded system, language learning, ERP, neural feedback

\section{Introduction}

Neural feedback in embedded system processing is an important branch of psycholinguistics. In the 1980s, Kutas and Hillyard first used ERP method to study the neural feedback mechanism of computer language processing [1]. At present, there are mainly the following kinds of EEG related to computer language processing: 1. Semanticrelated N400 [2] discovered by Kutas and Hillyard in the 1980s. 2. An ERP component related to programming program semantics, wherein P600 is associated with phrase components that do not conform to computer program semantics, and P600 reaches a peak approximately $500 \mathrm{~ms}$ after the acquisition of the relevant language phrase [3]. 3. Left anterior negative (LAN), which peaks about 300-500 ms after acquiring the relevant language phrase [4].

Language is a way of information continuity communication, and people usually complete the decomposition and integration of language information in instant or unconscious state. Therefore, language researchers, who want to understand the communication feedback mechanism of languages, need to explore how the language processing is carried out [5]. Meanwhile, the processing of computer language is clearly a function of the human brain [6], so the research on language processing is bound to study the connection between data and the brain. This paper mainly adopts 
ERP method to analyze the neural feedback mechanism of EEG signals in computer language processing. Such a study will not only involve a series of EEG signal fluctuation patterns related to language processing, but also have very important significance for the generation and fluctuation process of these EEG signals [7]. ERP method is the core part of studying neurocognitive linguistics, and under its impetus, nowadays, there are two different viewpoints about whether there is no influence, how to influence, and which processing method is used in the neural feedback of programming language processing and program semantic structure processing: sentencecentered theory and semantic-centered theory [8]. The sentence-centered theory holds that sentence processing and analysis is the main part of language processing while the semantic-centered theory holds that language processing is driven by semantics, and semantic information affects the semantic processing of sentence programs. Although sentence information has influence on semantics, it does not play a decisive role. The reason for the confrontation between the two main theories is that many researches lack the integration of program semantics and architecture semantics.

The purpose of this study is to explore the neural feedback of computer language learners on embedded system processing by using embedded system as experimental materials. It is necessary to determine whether the syntactic structure and semantics of embedded system have some influence on language processing, which provides more basis for the study of neural feedback in the process of embedded system. At the same time, it also provides new ideas for Chinese embedded system language teachers and computer language learners in the $\mathrm{PC}$ teaching research and learning process.

\section{Phrase Processing Experiment of Programming Language Based on EEG Signals}

\subsection{Experimental subjects}

Randomly recruit 20 paid college students as experimental subjects, all of whom passed CCT 3 and are right-handed, with normal vision or corrected vision, at the age of $18-20$ years old.

\subsection{Experimental materials}

Embedded system language is used as stimulating material. There are 30 groups of embedded system programs, among which 15 groups are CCT 3 high-frequency programs and 15 groups are CCT 3 low-frequency programs. There is no significant difference in program length.

\subsection{Experimental method}

When the brain performs language processing, the language is split and integrated in an instant, so the language research method should quickly and continuously detect the language processing process [9-10], and also be sensitive to the acquisition of 
language information without a subjective judgment of testers. It is the ERP method that best meets this requirement at present.

ERP is the accumulation of postsynaptic potentials produced by neurons in the brain, which reflects the response of EEG signals consistent with the time of stimulation of brain nerves and the recording of EEG signals produced in cognitive and other behavioral activities. ERP is mainly composed of such record indicators as positive and negative polarity, fluctuation amplitude and peak value, and EEG latency. Among them, the peak-peak value of EEG wave reflects the excitability of the brain, and the latency of EEG wave reflects the neural processing process and time after stimulation. ERP can record EEG signals in milliseconds, and can accurately record the generation and change of EEG signals [11-25].

\subsection{Experimental equipment}

The 64-lead ERP workstation manufactured by NeuroScan Company is used in the experiment. The electrodes are mounted according to international standards, and the fluctuation of experimental subjects' EEG signals during the experiment is recorded in real time by using the $\mathrm{Ag} / \mathrm{AgCL}$ electrode cap. Alternating current sampling (AC) is used, the filter bandpass is $0.01 \sim 50 \mathrm{~Hz}$, the sampling frequency is $400 \mathrm{~Hz} /$ conductor, and the scalp resistance is less than $5 \mathrm{k} \Omega$ [26]. The analysis period is $1000 \mathrm{~ms}$ after the presentation of the stimulus, and the baseline is $200 \mathrm{~ms}$ before the stimulation [27].

\subsection{Experimental procedure}

30 groups of embedded system program are randomly matched with different program semantics, among which 15 groups of embedded system program are consistent with program semantics and 15 groups of embedded system programs are inconsistent with program semantics. Subjects are asked to sit $60 \mathrm{~cm}$ in front of a computer screen, not to move their heads and to minimize blinking and other body movements during the experiment. The whole experiment process is completed by the subjects on computers. The computer screen will display 30 groups of embedded system program and the corresponding program semantics every 5 seconds in turn. The stimulus signals of embedded system program and semantics are presented with black characters in white background. The subjects only need to click on the Wrong key or Right key according to whether the semantics of embedded system program and program are matched. If it exceeds 5 seconds, it is regarded as Wrong and continues to present the next programming phrase stimulus signal. The experimental window is as shown in Figure 1: 


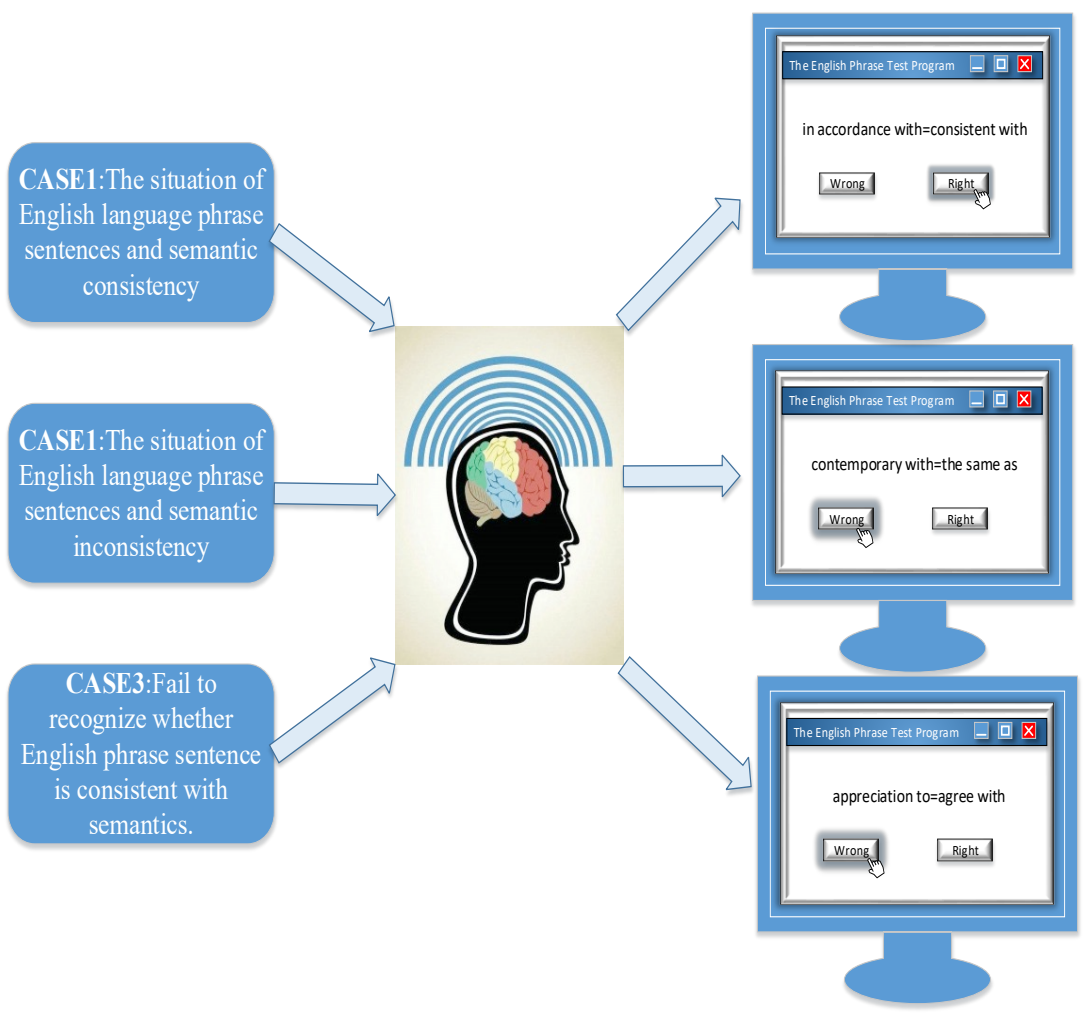

Fig. 1. English phrase test program

\subsection{Experimental data acquisition}

According to the general plan and predecessors' experience, the signal segmentation and baseline correction are considered comprehensively. Three points (F0, F1 and F2) are selected to collect EEG signals in the anterior part of the brain. $\mathrm{C} 0, \mathrm{C} 1$ and $\mathrm{C} 2$ are selected to collect EEG signals in the middle part of the brain. B0, B1, and B2 are selected to collect EEG signals in the posterior part of the brain. See Figure 2 for details. 


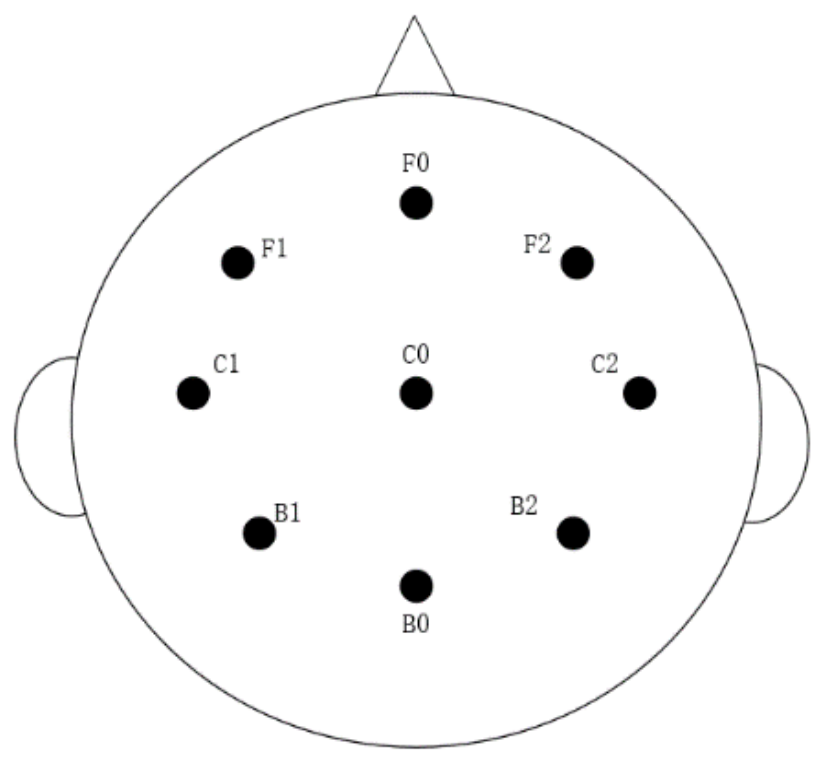

Fig. 2. EEG signal acquisition schematic

\section{Experimental Data Analysis}

3.1 ERP analysis of embedded system programs consistent with program semantics

When the embedded system program is consistent with the program semantics and the subjects are Right, N400 is found in the forehead and parietal part of the subject's brain, with a latency of about $500 \mathrm{~ms}$. We chose F0, F1, F2, and C0 for analysis, with the specific results shown in Figure 3.

One-way ANOVA shows that there are significant differences in the distribution of $\mathrm{N} 400$ in different brain regions (forehead, parietal, and posterior) $(\mathrm{F}(2,57)=3.473$, $\mathrm{p}=0.037$ ). By comparing the distribution of N400 in different parts of the brain, it is found that the distribution of N400 in the frontal and parietal parts of the brain is significantly greater than that in the posterior part of the brain $(p=0.014, p=0.038$, respectively), and there is no significant difference in the distribution of N400 between the frontal and parietal parts of the brain. 

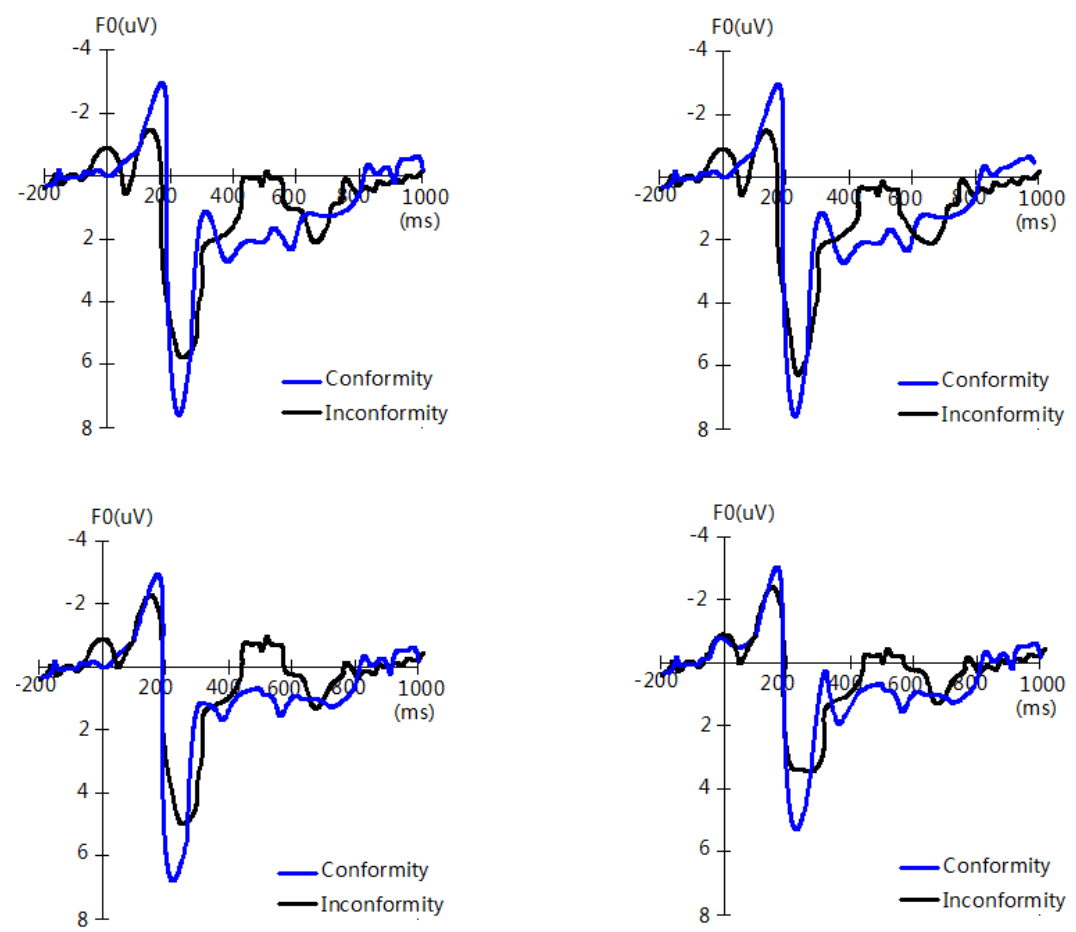

Fig. 3. ERP analysis of English language phrase semantics and sentence conformity

\subsection{ERP analysis of embedded system programs inconsistent with program} semantics

When the embedded system program is inconsistent with program semantics and the subjects are selected as Wrong, it's found that the ELAN (Early Negative Wave) with a latency of about $200 \mathrm{~ms}$ appears in the left forehead of the subjects, which gradually extends to the parietal part of the brain. At the same time, P600 (Late Positive Wave) with a latency of $500 \mathrm{~ms}-800 \mathrm{~ms}$ appears in the forehead and parietal parts of the brain. We chose $\mathrm{F} 0, \mathrm{C} 0, \mathrm{C} 1$, and $\mathrm{C} 2$ to analyze, and the result is shown in Figure 4 . 

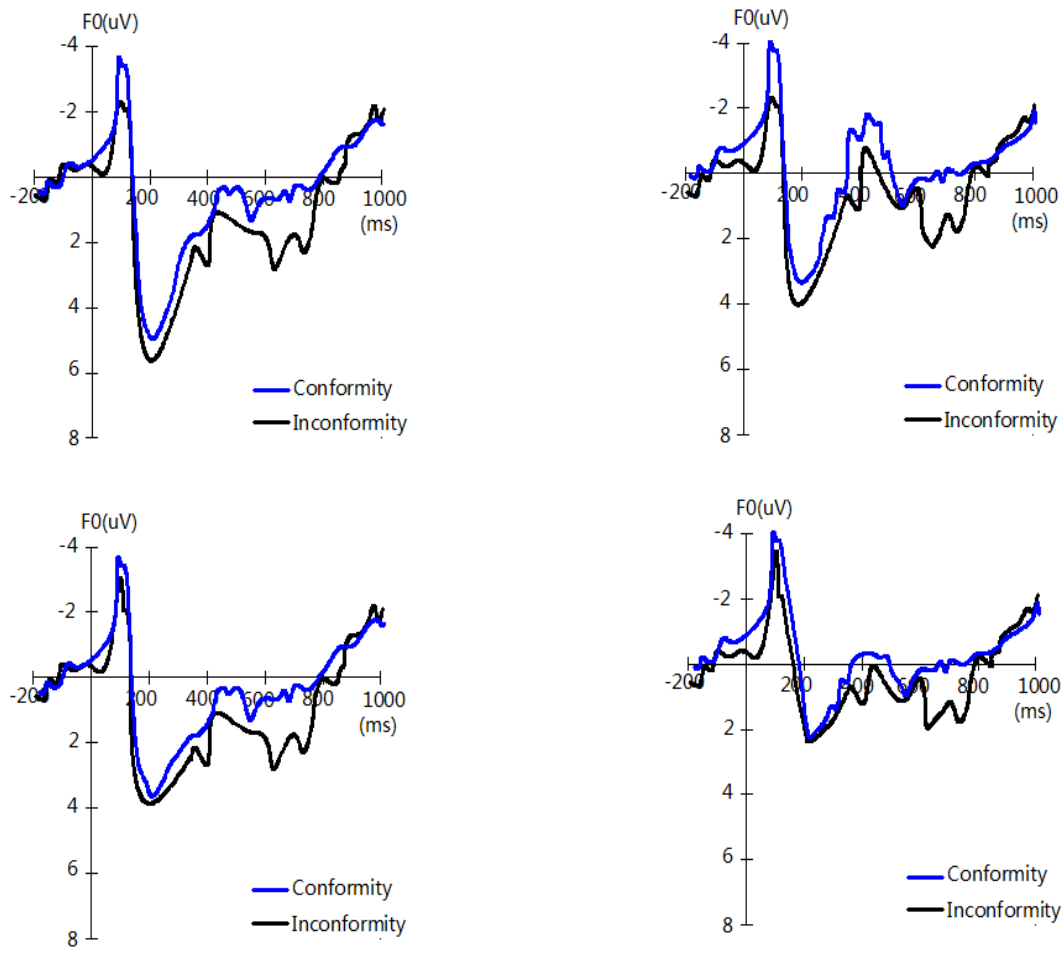

Fig. 4. ERP analysis of English language phrase semantics and sentence inconformity

There are significant differences in the distribution of ELAN waves in the brain (F $(2,57)=13.796, p=0.000)$. Compared with the right forehead and the parietal part of the brain, the amplitude of ELAN in the left forehead is the largest $(\mathrm{p}=0.029, \mathrm{P}=0$. 000 , respectively); the amplitude of ELAN produced with the consistency between embedded system program and program semantics is higher than that of ELAN produced with the inconsistency between embedded system program and program semantics $(\mathrm{t}(19)=-5.482, \mathrm{p}=0$. 000). There is also significant difference in the distribution of P600 in the cerebral cortex $(\mathrm{F}(2,57)=4.665, \mathrm{p}=0.012)$. In addition, comparatively, the distribution of P600 in frontal and parietal parts is significantly higher than that in posterior parts $(\mathrm{p}=0.019, \mathrm{p}=0.006$, respectively), but there is no significant difference between frontal and parietal parts of the brain $(p=0.676)$.

\subsection{ERP analysis of the failure of subjects to recognize the consistency of programming language phrases and sentences with semantics}

The experimental subjects' failure to identify the consistency between embedded system program and program semantics refers to when the presented embedded system program and program semantics are consistent and the experiment subjects are selected as Wrong or when the presented embedded system program and program 
semantics are inconsistent and the experiment subject are selected as Right, it's found with the average ERP waveform that P600 appears in the forehead and parietal parts of the brain with a latency of about $600 \mathrm{~ms}$; N400 is found in the forehead and parietal parts of the brain, with a latency of about $500 \mathrm{~ms}$. We chose F0, F1, C0, and C1 for analysis, and the specific results are as shown in Figure 5.
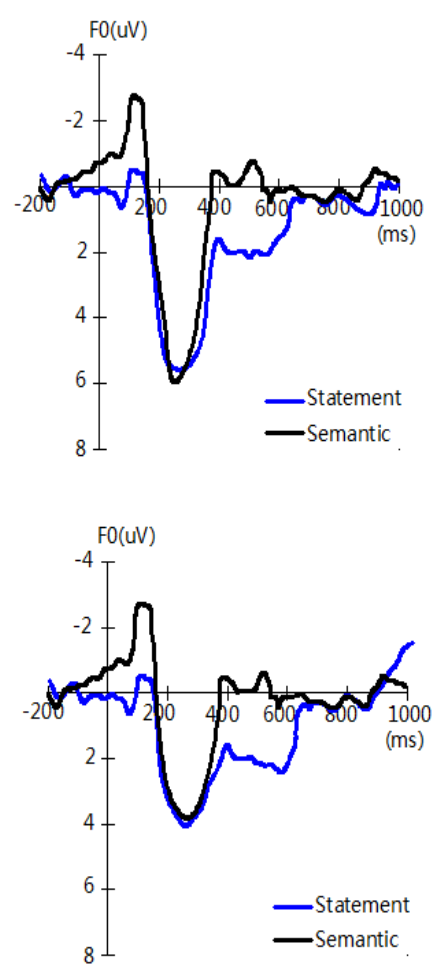
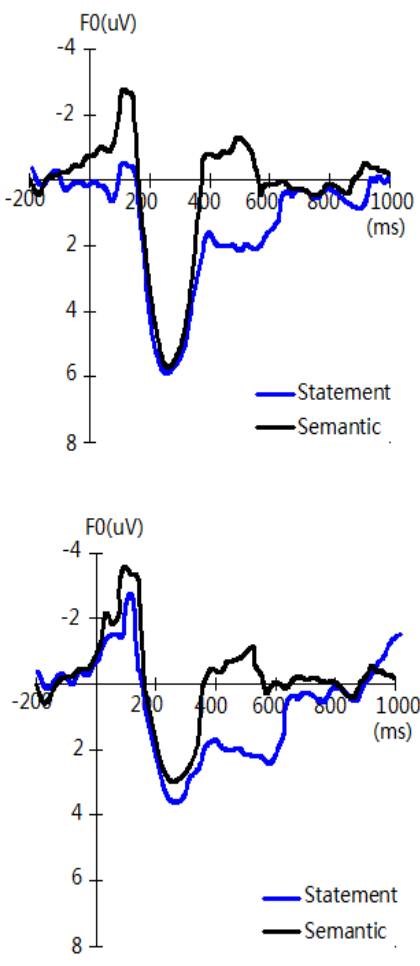

Fig. 5. ERP analysis of whether or not English phrasal phrases are consistent with semantics

\subsection{Comparison between the inconsistency between programming language phrases with semantics and the failure to recognize the consistency}

When the main effect of inconsistency between embedded system program and program semantics $(\mathrm{F}(1,76)=4.243, \mathrm{p}=0.043)$, and the main effect of brain region $(\mathrm{F}(1,76)=1.051, \mathrm{p}=0.821)$ are not significant, the interaction between inconsistency of sentence with semantics and brain $(F(1,76)=0.039, p=0.845)$ is not significant. From the average of the two cases, the amplitude of N400 caused by inconsistency of sentence and semantics is significantly larger than that caused by the failure to recognize the inconsistency $(2.54323>0.90641)$. When the main effect of embedded system program is inconsistent with program semantics $(F(1,114)=4.547, p=0$. 035) and the main effect of brain region are significant $(\mathrm{F}(2,144)=3.235, \mathrm{p}=0.043)$, the 
interaction between inconsistency of sentence with semantics and brain is not significant $(\mathrm{F}(2,114)=3.235, \mathrm{p}=0.043)$. The two-factor ANOVA of this case and the brain region shows that the amplitude of P600 produced with the inconsistency of sentence with semantics is significantly greater than that produced by the failure to recognize the inconsistency. The amplitudes of P600 in the forehead and parietal parts of the brain are significantly greater than those in the posterior part. The effect analysis on its interaction shows that the middle and parietal parts of the brain are significantly larger than the posterior part of the brain in the inconsistency of sentence with semantics $(\mathrm{F}(2,115)=7.58, \mathrm{p}=0.001)$; there is no significant difference in the forehead, parietal and posterior parts of the brain in the failure to recognize the inconsistency between the embedded system program and program semantics $(F(2,115)=0.07$, $\mathrm{p}=0.936$ ). There is a significant difference in $\mathrm{P} 600$ between the two stimuli in frontal and parietal parts of the brain $(\mathrm{F}(1,116)=4.72, \mathrm{p}=0.032) ;(\mathrm{F}(1,116)=7.14, \mathrm{p}=0$. $009)$, but no significant difference in posterior part of the brain $(\mathrm{F}(1,116)=1.19$, $\mathrm{p}=0$. 225). The comparison shows that the distribution of P600 in the parietal part of the brain is significantly greater than that in the posterior part of the brain $(p=0.019)$, but no significant difference is found between the anterior and posterior parts of the brain $(p=0.683)$ and between the anterior and posterior parts of the brain $(p=0.051)$, and there is no significant difference in the distribution of P600 in the brain without recognizing the consistency of programming language and semantics.

\section{Discussion}

The analysis of the above experimental data shows different waveform patterns of EEG signals triggered by the consistency and inconsistency of embedded system program and program semantics and failure to recognize the consistency. The negative wave of about $400 \mathrm{~ms}$ appears under the consistency between sentence and semantics, which is consistent with the previous discovery on N400. The early negative wave (ELAN) appears about $150 \mathrm{~ms}$ under the inconsistency between sentence and semantics and then the P600 (late positive wave) appears about $600 \mathrm{~ms}$. P600 is mainly distributed in the frontal and parietal parts of the brain, while ELAN is mainly distributed in the left frontal part of the brain, which is consistent with previous studies. The comparative analysis of the differences between N400 and P600 caused by the failure to recognize the consistency between sentences and semantics and the inconsistency between sentences and semantics shows that the amplitude change of $\mathrm{P} 600$ is larger than that of $\mathrm{N} 400$.

\section{Conclusion}

EEG signals ELAN and P600 are generated under the stimulation of the inconsistency between embedded system program and program semantics. EEG signals N400 are generated under the stimulation of the consistency between embedded system program and program semantics. EEG signals N400 and P600 are generated un- 
der the stimulation of the failure to recognize the consistency between embedded system program and program semantics. The conclusions are as follows:

1. Embedded system program and program processing have an important impact on the processing of program semantics. The difference between program processing and program semantics will result in different $\mathrm{b}$ neural signal feedback;

2. Embedded system program interacts with program semantics and plays a significant role in the process of program semantic processing.

3. In the embedded system program teaching and learning, the consideration of program processing and program semantics will be beneficial to the embedded system program teaching and learning, and the research of neural feedback signal provides a scientific basis for guiding the research on embedded system program processing.

\section{Acknowledgement}

This paper is a phased achievement of Jilin province "13th Five-Year Plan" Social science fund Project (2016B304).

\section{$7 \quad$ References}

[1] Zhou, D., Truran, M., Brailsford, T., Ashman, H. (2008). A hybrid technique for englishchinese cross language information retrieval. Acm Transactions on Asian Language Information Processing, 7(2), 1-35. https://doi.org/10.1145/1362782.1362784

[2] Ebert, U., Kirch, W. (2015). Scopolamine model of dementia: electroencephalogram findings and cognitive performance. European Journal of Clinical Investigation, 28(11), 944949. https://doi.org/10.1046/j.1365-2362.1998.00393.x

[3] Lockley, S. W., Evans, E. E., Scheer, F. A., Brainard, G. C., Czeisler, C. A., Aeschbach, D. (2006). Short-wavelength sensitivity for the direct effects of light on alertness. vigilance, and the waking electroencephalogram in humans. Sleep, 29(2), 161. https://doi.org/10.1093/sleep/29.2.161

[4] Loveless, N. E., Simpson, M., Näätänen, R. (2010). Frontal negative and parietal positive components of the slow wave dissociated. Psychophysiology, 24(3), 340-345. https://doi.org/10.1111/j.1469-8986.1987.tb00305.x

[5] Catani, M., Jones, D. K., \& Ffytche, D. H. (2005). Perisylvian language networks of the human brain. Annals of Neurology, 57(1), 8-16. https://doi.org/10.1002/ana.20319

[6] Huupponen, E., Maksimow, A., Lapinlampi, P., Särkelä, M., Saastamoinen, A., Snapir, A. (2010). Electroencephalogram spindle activity during dexmedetomidine sedation and physiological sleep. Acta Anaesthesiologica Scandinavica, 52(2), 289-294. https://doi.org/10.1111/j.1399-6576.2007.01537.x

[7] Craig, A., Tran, Y., Wijesuriya, N., Nguyen, H. (2012). Regional brain wave activity changes associated with fatigue. Psychophysiology, 49(4), 574-582. https://doi.org/10.1111/j.1469-8986.2011.01329.x

[8] Hardison, D. M. (2005). Variability in bimodal spoken language processing by native and nonnative speakers of english: a closer look at effects of speech style. Speech Communication, 46(1), 73-93. https://doi.org/10.1016/j.specom.2005.02.002 
[9] Shtyrov, Y., Smith, M. L., Horner, A. J., Henson, R., Nathan, P. J., Bullmore, E. T., et al. (2012). Attention to language: novel meg paradigm for registering involuntary language processing in the brain. Neuropsychologia, 50(11), 2605-2616. https://doi.org/10.1016/j.neuropsychologia.2012.07.012

[10] Shan, F. H., Zhao, L. Q., Yang, F. (2018). A novel semantic matching method for chatbots based on convolutional neural network and attention mechanism, Revue d'Intelligence Artificielle, 32(S1), 103-114. https://doi.org/10.3166/ria.32.s1.103-114

[11] Falkenstein, M. (2000). Erp components on reaction errors and their functional significance: a tutorial. Biological Psychology, 51(2), 87-107. https://doi.org/10.1016/s03010511(99)00031-9

[12] Wang, C. L., Wang, Q. Y., Cao, Y. P. (2017). Blind source separation algorithm for convolution mixed signals, Review of Computer Engineering Studies, 4(4), 103-107. https://doi.org/ 10.18280/rces.0400401

[13] Choubey, H., Pandey, A. (2018). Classification of healthy, inter-ictal and seizure signal using various classification techniques, Traitement du Signal, 35(1), 75-84. https://doi.org/10.3166/ts.35.75-84

[14] Nanova, P., Kolev, V., Yordanova, J. (2018). Effect of proactive mode of processing on event-related oscillatory brain responses in children, International Journal Bioautomation, 22(3), 253-262.

[15] Srikanth, B., Kumar, H., Rao, K. U. M. (2018). A robust approach for WSN localization for underground coal mine monitoring using improved RSSI technique, Mathematical Modelling of Engineering Problems, 5(3), 225-231. https://doi.org/10.18280/mmep.050314

[16] Peng, L. (2018). A brain nuclear magnetic resonance image segmentation algorithm based on non-rigid registration, Traitement du Signal, 35(3-4), 317-330. https://doi.org/10.3166/ts.35.317-330

[17] Zhao, X. (2018). Biomedical signal acquisition of hepatobiliary and portal vein before and after exercise, International Journal Bioautomation, 22(S4), 315-324. https://doi.org/10.7546/ijba.2018.22.4.315-324

[18] Shi, Y., Xiao, X. J., Lu, F. Q., Yang, X. F. (2018). Fingerprint positioning based on piecewise filtering of received signal strength indices and space-scene constraints, Review of Computer Engineering Studies, 5(2), 40-44. https://doi.org/10.18280/rces.050203

[19] Oostenveld, R., Praamstra, P. (2001). The five percent electrode system for high-resolution eeg and erp measurements. Clinical Neurophysiology, 112(4), 713-719. https://doi.org/10.1016/s1388-2457(00)00527-7

[20] Mihov, G. (2018). Subtraction procedure for power-line interference removal from ECG signals with high sampling rate, International Journal Bioautomation, 22(2), 147-158. https://doi.org/10.7546/ijba.2018.22.2.147-158

[21] Kumar, S., Yadava, M., Roy, P.P. (2019). Fusion of EEG response and sentiment analysis of products review to predict customer satisfaction, Information Fusion, 52, 41-52. https://doi.org/10.1016/j.inffus.2018.11.001

[22] O’Toole, J.M., Pavlidis, E., Korotchikova, I., Boylan, G.B., Stevenson, N.J. (2019). Temporal evolution of quantitative EEG within 3 days of birth in early preterm infants, Scientific Reports, 9(1), 4859. https://doi.org/10.1038/s41598-019-41227-9

[23] Abreu, R., Leal, A., Figueiredo, P. (2019). Identification of epileptic brain states by dynamic functional connectivity analysis of simultaneous EEG-fMRI: a dictionary learning approach, Scientific Reports, 9(1), 638. https://doi.org/10.1038/s41598-018-36976-y 
[24] Barik, K., Daimi, S.N., Jones, R., Bhattacharya, J., Saha, G. (2019). A machine learning approach to predict perceptual decisions: an insight into face pareidolia, Brain Informatics, 6(1), 2. https://doi.org/10.1186/s40708-019-0094-5

[25] Zhang, Y.Y., Palma, M.A., Jin, S., Yuan, X. (2019). US consumer reactions to China's Shuanghui acquisition of Smithfield Foods and its neural basis, Agribusiness, 35(1), 69-83. https://doi.org/10.1002/agr.21583

[26] Sánchez, P. J., Martínez, L., García-Martínez, C., Herrera, F., Herrera-Viedma, E. (2009). A fuzzy model to evaluate the suitability of installing an erp system. Information Sciences, 179(14), 2333-2341. https://doi.org/10.1016/i.ins.2008.12.020

[27] Wang, E., Chen, J. (2007). Effects of internal support and consultant quality on the consulting process and erp system quality. Decision Support Systems, 42(2), 1029-1041. https://doi.org/10.1016/j.dss.2005.08.005.

\section{Author}

Danbing Du She Earned her bachelor degree from Jilin University in 2003. Earned her master's degree from Jilin University of Finance and Economics in 2010. She areas of research interest include: Artificial intelligence \&education and New media technology teaching. She is a currently a lecturer of new media technology teaching at Changchun Normal University in education department.

Article submitted 2019-04-19. Resubmitted 2019-05-27. Final acceptance 2019-05-28. Final version published as submitted by the authors. 